\title{
The asynchronous discrete coding model: Further tests with single-attribute stimuli
}

\author{
JUDY ARNEL TREVENA and JEFF MILLER \\ University of Otago, Dunedin, New Zealand
}

\begin{abstract}
Two experiments using a probe-RT paradigm showed that partial information about the size of a stimulus can influence response processes before complete size information is available. Contrary to the asynchronous discrete coding model, these results suggest that the perceptual system may transmit to the response system information that only partially specifies a single stimulus attribute. In combination with previous findings, these results also suggest that there are at least two dissociable forms of selective preparation for a given response.
\end{abstract}

One of the most common assumptions about human information processing is that the flow of information through the nervous system can be decomposed into distinct stages (e.g., Donders, 1868/1969; Sternberg, 1969). For example, when the presentation of an object requires the performance of an action, the hypothetical stages may include stimulus detection, stimulus identification, selection of a response, response preparation, and response execution. Sternberg (1969) suggested that such stages occur in strict succession (i.e., in discrete stages), so that each stage can begin only after the previous stage has finished. Because the stages do not overlap, this conception predicts that the reaction time (RT) to a stimulus can simply be interpreted as the sum of the durations of the individual stages.

In contrast, other theorists have proposed continuous models in which stages ${ }^{1}$ may overlap in time (e.g., Eriksen $\&$ Schultz, 1979; McClelland, 1979). For example, suppose that the perceptual stage carries out a number of separate analyses on a given stimulus, and that as soon as the first analysis has been completed, its result is transmitted to the next stage. This next stage could then start processing based on the initial output from the perceptual stage, even though it would continue to receive more perceptual information as further discriminations were made. If these models are correct, response-level processing could begin before perceptual analysis completed, and RT could not be understood as simply the sum of the times for each of the processes required by the task.

As has been noted previously (e.g., Miller \& Hackley, 1992), neither discrete nor continuous models need be applicable under all circumstances, so the goal in studying these models is not so much to decide which one is true as to understand the conditions under which each

This research was supported by Otago Research Grant MFU-B75. The authors thank Michael Coles, Patricia Haden, Arthur Kramer, Robert Proctor, Rolf Ulrich, and an anonymous reviewer for helpful comments on earlier drafts of the article. Correspondence should be addressed to J. Miller, Department of Psychology, University of Otago, Dunedin, New Zealand (e-mail: miller@otago.ac.nz). model best describes the way we process information. For example, Meyer, Yantis, Osman, and Smith (1985) found evidence that processing tends to be discrete in tasks with few responses and compatible stimulus-response (S-R) mappings but tends to be continuous when there are many responses and incompatible $S-R$ mappings. In addition, Miller $(1982,1988)$ found evidence that processing tends to be discrete when the stimuli vary with respect to a single attribute. A further complication is that some pairs of successive stages may overlap, whereas other pairs may operate in strict sequence (Miller, 1988).

A number of researchers have looked particularly at the temporal relationship between the perceptual and response preparation stages (e.g., Coles, Gratton, Bashore, Eriksen, \& Donchin, 1985; De Jong, Wierda, Mulder, \& Mulder, 1988; Gratton, Coles, Sirevaag, Eriksen, \& Donchin, 1988; Miller, 1982, 1983, 1985, 1987; Miller \& Hackley, 1992; Mulder, Smid, \& Mulder, 1992; Osman, Bashore, Coles, Donchin, \& Meyer, 1992; Smid, Böcker, van Touw, Mulder, \& Brunia, 1996). According to discrete models, a stimulus must be completely identified before a response can be prepared, so there can be no temporal overlap between these two stages. According to continuous models, however, response preparation should, under some circumstances, be able to begin before stimulus identification is finished, in which case the stages would overlap temporally. In particular, there is evidence that multiple perceptual discriminations required to make a single response may be made at slightly different speeds (e.g., Miller \& Hackley, 1992; Posner, 1978), with some perceptual information being acquired relatively quickly and other information taking longer to obtain. If continuous models are correct, it should be possible for a response to be prepared as a result of partial output obtained from an early perceptual discrimination, even though additional perceptual analysis might be needed to determine the response uniquely. According to discrete models, on the other hand, the results of all perceptual discriminations become available to response preparation processes simultaneously, at the end of the stimulus identification stage, so no preliminary response preparation should occur. 
A variety of RT paradigms have been developed to test the contradictory predictions of discrete and continuous models regarding the temporal relationship of perception and response preparation (for reviews, see Meyer, Osman, Irwin, \& Yantis, 1988, and Miller, 1988), and further paradigms have been developed using a psychophysiological measure known as the lateralized readiness potential (LRP; e.g., Coles et al., 1985; De Jong et al., 1988; Gratton, Coles, \& Donchin, 1992; Miller \& Hackley, 1992; Osman et al., 1992; Smid et al., 1996). In general, the results obtained with these paradigms are in good agreement with one another, and they suggest an intermediate model. Evidence of preliminary response preparation has been found in experiments in which the stimuli have two or more relevant attributes. If early perceptual information uniquely specifies one stimulus attribute (e.g., red) but further perceptual analysis is needed to identify another relevant attribute (e.g., size), then response preparation can begin using the early information before perceptual analysis as a whole has finished (e.g., Miller \& Hackley, 1992; Smid et al., 1996). In contrast, evidence of preliminary response preparation has not been found in experiments with stimuli varying along a single attribute (e.g., size). For these experiments, stimuli are chosen so that early perceptual information partially specifies the attribute (e.g., larger than average) but further perceptual analysis is needed to determine a unique value of the attribute (e.g., largest vs. second largest). Moreover, the difference between single-attribute and multiattribute stimuli does not seem to be an artifact of differences in discriminability, because discriminability differences were comparable in the two different types of stimulus sets (Miller, 1982).

Miller (1982) proposed the asynchronous discrete coding (ADC) model to account for this difference between single-attribute and multiattribute stimuli. According to the ADC model, the perceptual stage discretely outputs information in codes corresponding to fully processed stimulus attributes. Thus, it is possible for response processes to receive preliminary perceptual information only when stimuli are coded using two or more attributes, not when they are coded in terms of a single attribute. This explains the effects of attributes on preliminary response preparation, because such preparation can occur only when response processes receive the appropriate preliminary information.

Although the ADC model provides an attractive explanation of the difference in preliminary response preparation for single-attribute versus multiattribute stimuli, three recent studies provide reasons to doubt this model. Using three different tasks, Band and Miller (1997), Ilan and Miller (1998), and Miller, Coles, and Chakraborty (1996) all tested for effects of preliminary information using both the LRP and a probe-RT measure described in Experiment 1. All three studies found a dissociation between these two measures: probe RTs were influenced by preliminary information, but LRPs were not. Moreover, Miller et al. (1996) found this dissociation within a single data set (i.e., same subjects and experimental trials), ruling out the possibility that subtle changes in experimental procedure were responsible for differences in the two measures. One plausible interpretation of this dissociation is that the probe RT and LRP paradigms are sensitive to different kinds of effects of preliminary information. More specifically, Miller et al. (1996) argued that the probe RT must measure an effect of preliminary information on a response-related stage preceding response preparation (e.g., response selection), whereas the LRP measures an effect on more peripheral motor preparation processes. Another possible interpretation of the dissociation is that the probe-RT measure is simply more sensitive than the LRP, but this interpretation is implausible for reasons that will be presented in the General Discussion section.

The dissociation between different response-related effects of preliminary information raises doubt about the ADC model, because it highlights the possibility that previous studies with single-attribute stimuli might have missed an effect of preliminary information on a responserelated stage prior to preparation. Such studies tested for effects of preliminary information with the LRP and other measures of activity at the response preparation stage, but no previous study of single-attribute stimuli has used the probe-RT task, which appears to be sensitive to earlier response-related stages. According to the ADC model, preliminary information about single-attribute stimuli should not affect any response-related stage, because such information is never transmitted out of the perceptual process. Thus, the ADC model predicts that preliminary information about single-attribute stimuli should have no effect in the probe-RT task, just as it has no effect on the LRP. The present experiments tested this prediction by looking for effects of preliminary information about single-attribute stimuli in the probe-RT task.

\section{EXPERIMENT 1}

The stimuli used in Experiment 1 were four squares varying in the single attribute of size (approximately 8 , 10,16 , and $20 \mathrm{~mm}$ on a side), as used in two previous studies finding no evidence of preliminary response preparation (Miller, 1983; Miller \& Hackley, 1992). In each trial, one square was presented at the fixation point. The largest and smallest squares were targets, and each required a response with the left or the right hand. The two squares of intermediate sizes were distractors, and they did not require a response.

With these stimuli, the perceptual system should have preliminary information about size before it has complete information, because each of the two distractor squares was quite similar in size to one of the target squares. For example, when the 16-mm distractor is presented, perceptual analysis should reveal relatively rapidly that one of the two larger squares has been presented, but further analysis would be needed to determine whether the stimulus was the larger target or the larger distractor. (A pilot 
study reported by Miller \& Hackley, 1992, supported the hypothesis that, for these squares, discriminations between the large and the small pairs are faster than discriminations between the members of a pair.) Continuous models predict that this preliminary size information would be transmitted to the response preparation process, where it would cause selective preparation of the response hand consistent with the preliminary information (i.e., the response hand to the largest target square is assigned). Miller (1983) and Miller and Hackley, however, found no evidence of response preparation in two previously developed paradigms, and this was interpreted as support for the ADC model's assumption that partial information about the size attribute was never transmitted out of the perceptual stage.

The present probe-RT paradigm uses a divided attention task as another way to test for a response-level effect of preliminary information about size (cf. Miller, 1985, 1987). Shortly after the appearance of a distractor square, a tone was sometimes presented in one ear, and the subject was instructed to respond immediately to any tone with the ipsilateral hand. RTs to the tones can be used to detect response-level effects caused by the distractor. When the distractor is the second-largest square, for example, preliminary processing at the response level, if it takes place, would be expected to facilitate or prime the response assigned to the largest square target. In the presence of this distractor, then, a response to a tone should be especially fast if the tone requires the response assigned to the largest square, and it should be especially slow if the tone requires the competing response. Analogously, when the distractor is the second-smallest square, a response to a tone should be especially fast if the tone requires the response assigned to the smallest square. Thus, the comparison of interest is between trials in which the tone requires the response primed by the distractor ("consistent trials") and trials in which the tone requires the opposite response ("inconsistent trials"). If responses are faster on consistent trials than on inconsistent trials, then we can conclude that preliminary size information causes some processing at the response level, contrary to the prediction of the ADC model.

Another factor that must be manipulated within the probe-RT paradigm is the time interval between the onset of the square and the onset of the tone, termed the stimulus onset asynchrony (SOA). Even if the distractor square does cause some processing at the response level, this effect should be detectable only in probe RTs at medium SOAs. If the SOA is too short (when the tone is presented very early), the distractor square will not yet have had an opportunity to influence response processes, so no consistency effect will be found. If the SOA is too long (when the tone is presented very late), the distractor square's influence on response processes may have faded away following recognition that the square was indeed a distractor, as do effects of other types of irrelevant information (e.g., Flowers, 1990; Hommel, 1993), so again a consistency effect need not be found. This analy- sis suggests that the consistency effect should be largest at an intermediate SOA, probably just before the point at which the square is recognized as a distractor. Since it is not clear exactly what numerical value this SOA should have, we used a range of SOAs $(350,500$, and $650 \mathrm{msec})$ chosen to cover the RT distribution obtained in this task, thereby spanning the range at which the consistency effect seemed most likely to be present.

\section{Method}

Subjects. A total of 36 first-year students from the University of Otago participated in a single 40-min experimental session, as partial fulfillment of a course requirement.

Apparatus and Stimuli. The presentation of stimuli and recording of responses and RTs were controlled by an IBM-PC-compatible computer. Squares were presented as bright line figures against the dark background of the computer monitor, with lines approximately $1 \mathrm{~mm}$ thick. In each trial, one of the four possible squares was presented at fixation in the center of the computer monitor. The subjects viewed the visual stimuli from a distance of approximately $75 \mathrm{~cm}$, so the squares ranged in size from approximately $0.6^{\circ}$ to $1.5^{\circ}$ of visual angle on a side. The auditory stimuli were clearly audible 56 -msec tones, approximately $50 \mathrm{~dB}$ in intensity, presented to either the left or the right ear via headphones, with SOAs of 350,500 , or $650 \mathrm{msec}$, relative to the onset of the squares.

The subjects were instructed to respond to any target square (small or large) or to any target tone (left or right ear) with the left or the right hand, using the S-R assignments shown in Table 1. In brief, the subjects responded to the largest square with one hand and the smallest with the other hand, but they were not to respond to the second-largest or second-smallest squares. A tone in either ear required a response by the hand on the same side. The assignment of largest versus smallest square to the left or right hand was counterbalanced across subjects.

Each response consisted of a series of three keypresses in the order index finger, ring finger, and middle finger. We used this three-keypress response mainly for compatibility with a previous study using the LRP to test for response preparation with these same stimuli (Miller \& Hackley, 1992); it was used in the earlier study because it had been

Table 1

Conditions and Numbers of Trials per Block in Each Condition in Experiments 1 and 2

\begin{tabular}{lllcc}
\hline \multicolumn{1}{c}{ Stimuli } & & Correct & \multicolumn{2}{c}{ Number of Trials } \\
\cline { 4 - 5 } Size of Square & Tone & Response & Experiment 1 & Experiment 2 \\
\hline \multicolumn{5}{c}{ Visual Target Conditions } \\
Smallest & None & Left & 12 & 32 \\
Largest & None & Right & 12 & 32 \\
\multicolumn{5}{c}{ No-Go Conditions } \\
2nd smallest & None & No-Go & 12 & \\
2nd largest & None & No-Go & 12 \\
\multicolumn{5}{c}{ Nontarget Squares, Consistent Tone Targets } \\
2nd smallest & Left & Left & 6 & 4 \\
2nd largest & Right & Right & 6 & 4 \\
\multicolumn{5}{c}{ Nontarget Squares, Inconsistent Tone Targets } \\
2nd smallest & Right & Right & 6 & 12 \\
2nd largest & Left & Left & 6 & 12 \\
\hline
\end{tabular}

Note-This table reflects the stimulus-response mapping used for half of the subjects. For the other half, the responses "left" and "right" were interchanged. In conditions with both a square and a tone stimulus, trials were divided equaliy among the stimulus onset asynchronies used in each experiment. 
found to maximize preparatory LRP (Hackley \& Miller, 1995). Responses were made on a standard computer keyboard, with these three fingers of each hand positioned at each end of the bottom row in the most natural positions. Thus, the left-hand response consisted of pressing the $\mathrm{C}, \mathrm{Z}$, and $\mathrm{X}$ keys, in that order, and the right-hand response consisted of pressing the comma, slash, and period keys.

Procedure. Each session consisted of six blocks of 72 trials each, with the number of trials divided across conditions as shown in Table 1. Within each condition with both a square and a tone, the trials were divided equally across SOAs.

Each trial began with a fixation cross, shown for $800 \mathrm{msec}$ in the center of the screen. After fixation offset, there was an interval of $700 \mathrm{msec}$ before a square appeared at the center of the screen, and it remained until the subject responded or the trial terminated. On trials with tones, the tone was presented starting at the appropriate SOA after the onset of the square. RT was measured from the onset of the target square or tone, whichever was presented on the trial, to the first keypress of the response. The trial terminated $2 \mathrm{sec}$ after the presentation of the target stimulus (target square or tone) or $2 \mathrm{sec}$ after the presentation of the nontarget square in no-go trials, implying that the maximum RT was 2,000 msec. Feedback was provided after each trial, in the form of the word correct $3.5 \mathrm{~cm}$ above the fixation point for $600 \mathrm{msec}$ or error $3.5 \mathrm{~cm}$ below the fixation point for $1,200 \mathrm{msec}$, as appropriate. The next trial began approximately $1 \mathrm{sec}$ after the end of the feedback.

\section{Results and Discussion}

Mean RT was somewhat elevated in the first block but was fairly consistent over the remaining five blocks. The first block was therefore considered practice and was excluded from the analysis. Incorrect trials and trials with RTs less than $200 \mathrm{msec}$ were also excluded from the analysis.

No-go trials. Overall, the subjects correctly withheld the response on $93.2 \%$ of no-go trials (i.e., a visual nontarget square without a tone), but they made false alarms on the other $6.8 \%$. False alarms were virtually always responses appropriate to the more similar target square (6.7\% of the $6.8 \%$ ). Thus, the false alarms made on no-go trials could well have resulted from perceptual misidentifications of a nontarget square as being the target that was more similar in size. False alarms were more likely with the second-smallest square $(9.6 \%)$ than with the second-largest one $(3.9 \%)\left[F(1,34)=14.4, M S_{\mathrm{e}}=39.7\right.$, $p<.01]$, suggesting that either the discrimination between the smaller two squares was especially difficult or there was a bias to respond to small squares.

Responses to target squares. Across all subjects, only 1 of the 4,320 trials with visual target squares was eliminated because of an RT less than $200 \mathrm{msec}$. Responses to target squares were correct on $95 \%$ of trials, and these correct responses had an average RT of $714 \mathrm{msec}$. Responses to small target squares were faster and more accurate than responses to large ones [RT, $698 \mathrm{vs.} 730 \mathrm{msec}$, $F(1,34)=4.5, M S_{\mathrm{e}}=4,099, p<.05$; percent correct, $96.0 \%$ vs. $\left.93.9 \%, F(1,34)=10.3, M S_{\mathrm{e}}=20.98, p<.01\right]$; together with the high rate of false alarms to small nontarget squares, this suggests that there was a particular bias to respond to small squares. Of the $5 \%$ errors to target squares, $4.7 \%$ were failures to respond, indicating a confusion with a nontarget square, and the other $0.3 \%$ were responses with the incorrect hand or with an incorrect key on the correct hand.

Responses to probe tones. Figure 1 shows the frequency distributions of RTs to probe tones, separately for each SOA, pooling across subjects and consistent versus inconsistent trials. As is evident in the figure, nonnegligible proportions of trials were eliminated because of RTs less than $200 \mathrm{msec}$ at the two longer SOAs. Indeed, at these SOAs there were even responses before probe onset (i.e., RTs less than zero). These results suggest that the subjects occasionally made false-alarm responses to nontarget squares, producing responses that were too fast to be generated in response to the tones. Interestingly, the frequency distributions at the two larger SOAs also show bimodality, with especially few responses in the interval 150-200 msec after tone onset. This suggests that the onset of the tone shuts down the process generating false alarms; if this process has not already generated a false alarm when the tone occurs, it will never do so. As discussed further later, these distributions thus suggest that a 200-msec-lower RT cutoff is appropriate for eliminating false alarms made to the squares.

Figure 2 shows mean correct RTs to tone probes, averaged over subjects, groups, hands, and blocks. On average, these were $26 \mathrm{msec}$ faster in consistent trials than inconsistent trials, with mean RTs of 490 and $516 \mathrm{msec}$, respectively.

A $2 \times 2 \times 3 \times 2$ analysis of variance (ANOVA) of probe RT, with factors of response hand (left or right), consistency (consistent or inconsistent), SOA $(350,500$, or $650 \mathrm{msec}$ ), and group (whether the response to the smallest square was assigned to the left or right hand) confirmed the significance of the consistency effect $\left[F(1,34)=24, M S_{\mathrm{e}}=3,088, p<.01\right]$. The hand $\times$ consistency interaction was the only other significant effect in this analysis, with a greater consistency effect for responses made with the left hand $(34 \mathrm{msec})$ than for those made with the right hand $(18 \mathrm{msec})[F(1,34)=4.38$, $\left.M S_{\mathrm{e}}=1,638, p<.05\right]$. In an analogous ANOVA on percent correct, the only significant effect was the hand $X$ SOA interaction $\left[F(2,68)=4.86, M S_{\mathrm{e}}=6.05, p<.02\right]$, reflecting higher accuracy for the right hand at the two shortest SOAs but higher accuracy for the left hand at the longest SOA. This interaction is not necessarily meaningful, however, because it could have been due to the nonlinear scaling properties of percent correct (Loftus, 1978).

The consistency effect obtained in Experiment 1 is evidence that preliminary information about size has an effect on response-level processes and, hence, that the perceptual process transmits partial size information before the size attribute has been fully identified. Clearly, this finding is evidence against the ADC model's basic assumption that the perceptual system transmits information only about fully identified attributes. Of course, the present finding is consistent with models postulating continuous transmission of information from the perceptual process to response processes. As noted earlier, however. continuous models have difficulty explaining the absence 

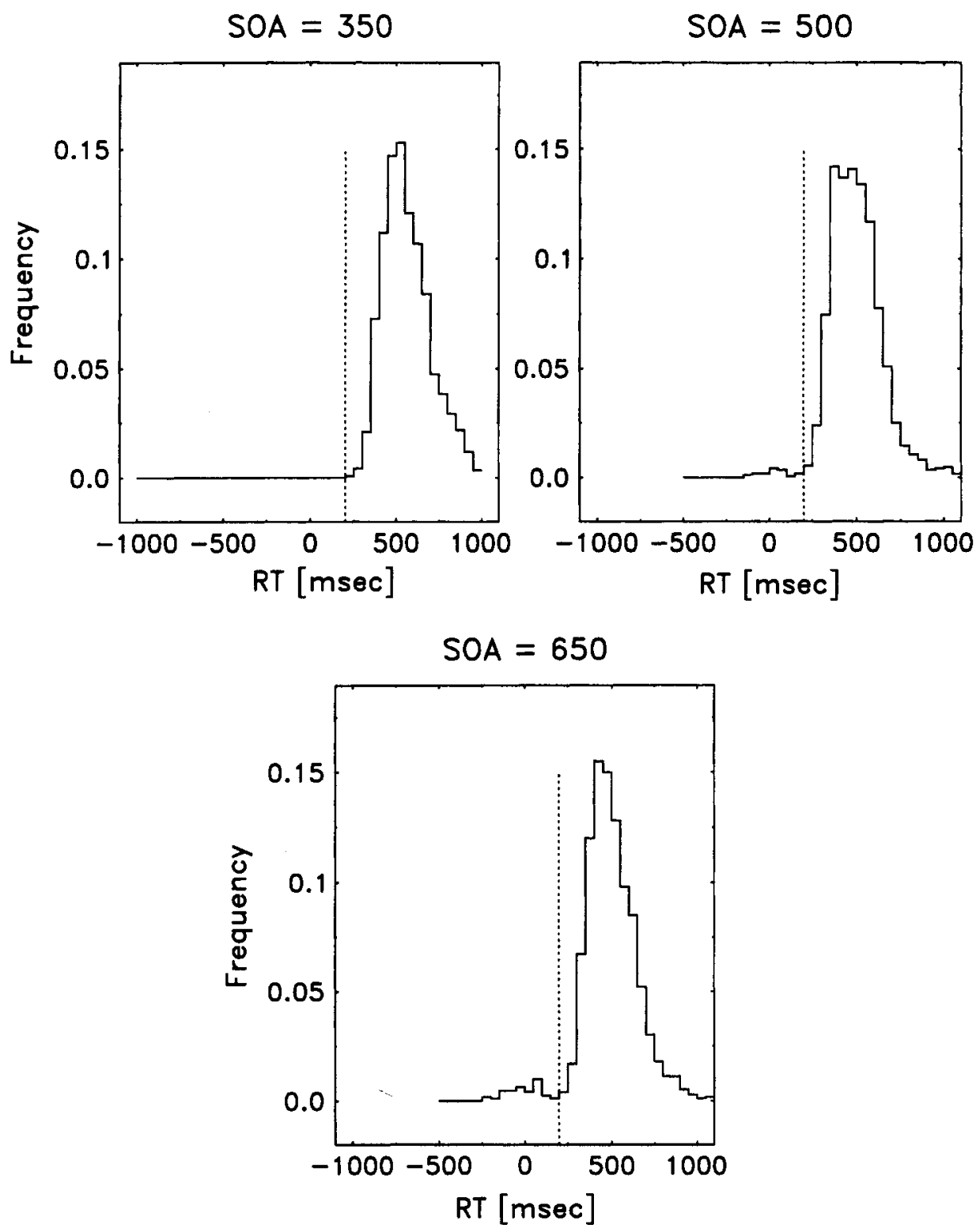

Figure 1. Observed frequency distributions of reaction times (RT) to probe tones at each stimulus onset asynchrony (SOA) in Experiment 1, pooling together trials with consistent and inconsistent probe tones (solid lines). Negative RTs indicate responses before tone onset. The dotted vertical line shows the 200-msec RT cutoff; responses faster than this cutoff were excluded in comparing the consistent and inconsistent conditions.

of response preparation effects observed when preliminary information partially, rather than fully, specifies an attribute, as with the stimuli varying in size used here (Miller, 1982, 1983; Miller \& Hackley, 1992). We will consider further, in the General Discussion section, what sort of model might accommodate the full pattern of results.

Although it seems plausible to explain the consistency effect in terms of preliminary response preparation, it is necessary to consider another alternative artifactual explanation of the effect. ${ }^{2}$ Suppose that subjects sometimes make fast false-alarm responses to no-go squares, re- sponding to them as if they were targets of the similar size. These fast responses would be correct-and therefore included in the analysis-for the consistent condition but not for the inconsistent one. The consistent condition's mean RT would thus be artifactually reduced, relative to the inconsistent condition's, by the inclusion of these fast false-alarm responses. Three aspects of the data rule out this alternative explanation. First, Figure 3 shows vincentized cumulative distribution functions (CDFs) of RT for the consistent versus inconsistent condition at each SOA. If the difference in means were due to 


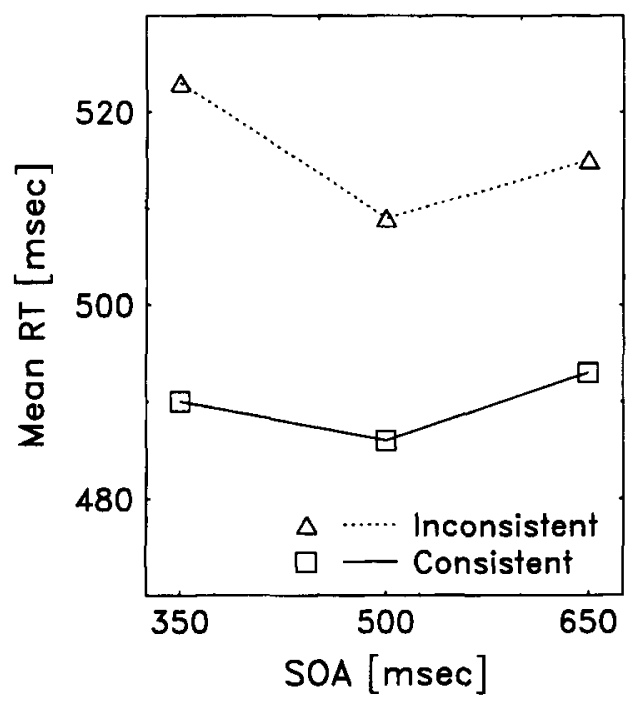

Figure 2. Mean reaction time (RT) to probe tones as a function of square consistency and square-to-tone stimulus onset asynchrony (SOA) in Experiment 1.

the inclusion of a few fast false alarms only in the consistent condition, the CDFs should differ substantially at the lowest percentiles but should converge. Contrary to this prediction, Figure 3 shows that responses were faster in the consistent condition than in the inconsistent one throughout virtually the full range of the RT distributions. Second, the consistency effect is still highly significant $(p<.01)$ and in fact numerically slightly larger $(30 \mathrm{msec})$ if error trials are included in the analysis. Including errors would allow inconsistent trials to benefit from the hypothesized fast false alarms just as much as consistent trials and thus would eliminate the consistency effect if this artifact produced it. Thus, this analysis shows that the consistency effect is still strong even if the biasing effects of fast guesses are allowed to contribute equally to consistent and inconsistent trials. Third, as discussed earlier in connection with Figure 1, the frequency distributions of RTs to probe tones suggest that the lower cutoff of $200 \mathrm{msec}$ was effective in excluding all false alarms from the analyses of the means and CDFs of RT.

Numerically, the consistency effect decreased monotonically across SOAs $(33,23$, and $22 \mathrm{msec}$ at SOAs of 350,500 , and $650 \mathrm{msec}$, respectively), but neither the consistency $\times$ SOA interaction nor its linear component was statistically reliable. One extremely problematic interpretation of this pattern is that the consistency effect does not reflect partial information after all, but, instead, it arises after complete perceptual analysis of the square's size. On this interpretation, the similarity in size of a known nontarget to one particular target might prime the response associated with that target. This interpretation seems somewhat unlikely because the consistency effect develops so quickly that it is already strong at an SOA of $350 \mathrm{msec}$. Given that the mean RT to visual targets was
$714 \mathrm{msec}$, it seems unlikely that subjects would have finished identification of a nontarget size by the time they had generated a response to a probe tone at that SOA. Given the importance of this "complete information" hypothesis, however, Experiment 2 was a further test of it.

A second possible explanation for the insensitivity of the consistency effect to SOA is that the expected interaction was removed by averaging across subjects who showed the consistency effect at different SOAs. To evaluate this explanation, we conducted an ANOVA treating subjects as an experimental (i.e., fixed-effects) factor and using block-to-block variation within a subject and condition to estimate error variance. This analysis indicated that the SOA $\times$ consistency interaction did not vary significantly from one subject to the next $(F<1)$.

Finally, a third possible explanation for the absence of a consistency $\times$ SOA interaction is that the consistency effect is fully developed by the shortest SOA included in the experiment $(350 \mathrm{msec})$ and that it is long lasting relative to the present range of SOAs. In principle, the effect might be long lasting either because recognition of the square as a distractor takes quite a long time or because the consistency effect fades slowly once the square is recognized to be a distractor. Given that approximately $50 \%$ of all RTs to target squares were less than the longest $\mathrm{SOA}(650 \mathrm{msec})$, however, it seems likely that recognition of a square as a distractor must often be completed by the longest SOA. Thus, the available data appear to favor the explanation that the consistency effect fades slowly relative to the present range of SOAs. Experiment 2 included a larger range of SOAs to look at this interaction in more detail.

In addition to providing evidence of response processing using partial information about size, the present results also provide further evidence that there are dissociations among different types of response processes (cf. Miller et al., 1996). Specifically, the present evidence that partial information about size can cause response processing of the sort measured by the probe-RT task conflicts with previous evidence indicating that it does not cause more peripheral response preparation of the sort measured in other paradigms (e.g., Miller \& Hackley, 1992). The most obvious explanation of this pattern of results is that there are at least two dissociable types of response processing, in which case multiple measures are clearly needed to investigate the influence of partial information on responses.

\section{EXPERIMENT 2}

The consistency effect demonstrated in Experiment 1 suggests that, contrary to the ADC model, partial information about stimulus size can be used for at least some response-level processing before complete size information is available. This conclusion is weakened, however, by the absence of the predicted interaction between consistency and SOA. To provide more evidence that the 

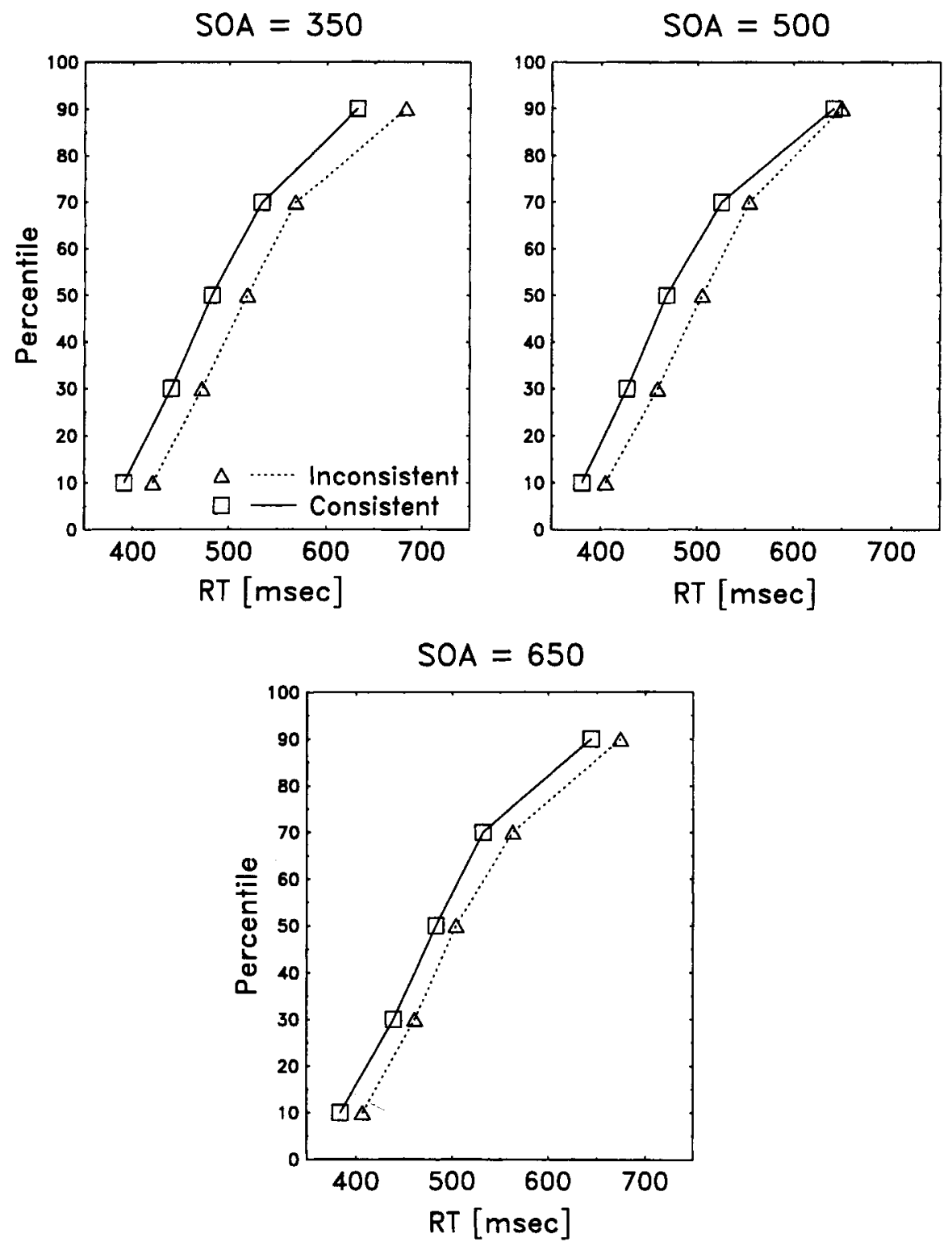

Figure 3. Vincentized cumulative distribution functions showing the mean reaction time (RT) to probe tones at each of five percentiles for consistent and inconsistent conditions at each stimulus onset asynchrony (SOA) in Experiment 1.

consistency effect does occur before complete information about size is available, it is necessary to show that the effect dissipates at long SOAs.

Experiment 2 was a replication of Experiment 1, with two changes that were expected to increase the consistency $\times$ SOA interaction if the consistency effect is indeed produced by partial information about size. First, it used a wider range of SOAs $(100,350,700$, and $1,200 \mathrm{msec})$ to provide a stronger test for interaction by monitoring the onset and decay of preparation over a longer time interval. The range of SOAs (350-650 msec) used in Experiment 1 might have been too narrow to see a clear change in the consistency effect.
Second, as shown in Table 1, Experiment 2 included three times as many inconsistent trials as consistent ones. The preponderance of inconsistent trials was used so that partial and full information would favor opposite responses. Partial information favored the consistent response, as in Experiment 1. Given partial information that the visual stimulus was one of the smaller two squares, for example, there was a $75 \%$ chance that the consistent left-hand response would be required (i.e., $[32+4] /$ $[32+4+12])$. Full information about a visual nontarget, however, actually favored the inconsistent response. Given complete information that the visual stimulus was specifically the second-smallest square, for example, there 
was a $75 \%$ chance that the inconsistent right-hand response would be required (i.e., 12/[4+12]).

Given that partial and full information favored opposite responses, an even stronger interaction of consistency and SOA would be predicted if partial information does indeed cause a consistency effect. Partial information would favor the consistent response, as in Experiment 1, leading to a consistency effect at short-to-medium SOAs. Once full information was available, however, the preponderance of inconsistent trials gives the subject an incentive to undo the initial response-level processing, thus reducing and possibly reversing the consistency effect at long SOAs.

Quite a different result would be predicted if the consistency effect arises after size analysis is finished, however, as asserted by the problematic "complete information" interpretation of Experiment 1. According to this interpretation, there should be no consistency effect at short SOAs, because full information is not yet available to influence response processes. At longer SOAs, full information is available, and the inconsistent response should be favored because of the preponderance of inconsistent trials. However, there should be no SOA at which consistent trials are faster than inconsistent ones; therefore, there should at no SOA be a positive consistency effect as observed in Experiment 1.

\section{Method}

The subjects were 31 students recruited from the same pool as those used in Experiment 1; one was excluded because of an excessive error rate. The stimuli, apparatus, and procedure were the same as those used in Experiment 1, except as follows: There were six blocks of 96 trials for each subject. As shown in Table 1, each block included 64 trials with visual targets ( 32 of each size; i.e., requiring left- vs. right-hand responses). It also included 32 nontarget squares: 8 were accompanied by consistent tones ( 1 per SOA for each response hand), and 24 were accompanied by inconsistent tones ( 3 per SOA for each response hand). No-go trials were omitted from this experiment in order to increase the number of trials in the conditions of interest. The SOA from square onset to tone onset was 100, 350, 700 , or $1,200 \mathrm{msec}$, with these SOAs tested equally often.

\section{Results and Discussion}

As in Experiment 1, the first block was considered practice and was not included in the analysis. Incorrect trials and trials with RTs less than $200 \mathrm{msec}$ were also excluded from the analysis.

Responses to target squares. Across all subjects, only 2 of the 9,600 trials with target squares were excluded because of RTs less than $200 \mathrm{msec}$. Correct responses to target squares (98.1\%) had an average RT of $749 \mathrm{msec}$. Responses to small target squares were significantly faster than responses to large ones [ $713 \mathrm{vs.} 785 \mathrm{msec}, F(1,28)=$ $\left.16.5, M S_{\mathrm{e}}=4,732, p<.01\right]$; they were also $0.7 \%$ more accurate, but this difference only approached significance $(p<.10)$.

Responses to probe tones. A preliminary examination of RTs to tones revealed that the subjects made a substantial number of false alarms to nontarget squares. Fig- ure 4 shows the frequency distributions of observed RTs at each SOA, pooling together consistent and inconsistent trials. Many of the RTs were negative, especially at the longer SOAs, indicating that the subjects often responded even before tone onset. Clearly, these responses must have been false alarms. Table 2 shows that the falsealarm responses were almost all consistent with the early information about size, as in Experiment 1, which indicates that they probably resulted from perceptual misidentifications of the nontarget size as the more similar target size. The false-alarm rate in Experiment 2 was considerably higher than that in Experiment 1, however, especially at the longer SOAs. It seems plausible that the absence of no-go trials in this experiment caused the subjects to relax the criterion for accepting a square as a match to a target. Moreover, the increase in false alarms over SOAs suggests that the subjects continually reevaluated the stimulus as a possible target, often accepting it as a target even after it had initially been classified as a nontarget (see Levy \& Pashler, 1995). Most importantly, however, the frequency distributions at all four SOAs suggest that a 200-msec-lower cutoff on RTs would successfully eliminate the false-alarm responses from the analysis, so that the means of the remaining consistent and inconsistent trials would not be contaminated by these false alarms. As in Experiment 1, the frequency distributions reveal a clear disappearance of false alarms in the range $150-200 \mathrm{msec}$ after tone onset, again suggesting that tone detection terminates the false-alarm process.

For each subject, mean RTs to tone probes were computed as a function of SOA and consistency, averaging across blocks and hands and excluding errors and trials with RTs less than $200 \mathrm{msec}$. Figure 5 shows the means of these values across subjects. The individual subject means and corresponding percents correct were entered into ANOVAs with factors of group (response hand to which the smallest square was assigned), SOA, and consistency. The analysis of RTs yielded only three significant sources of variance, all of which are apparent in Figure 5: (1) RT decreased as SOA increased $[F(3,84)=$ $\left.167, M S_{\mathrm{e}}=1,736, p<.001\right]$, (2) responses were faster in consistent trials than in inconsistent ones [464 vs. $\left.489 \mathrm{msec} ; F(1,28)=20.7, M S_{\mathrm{e}}=1,719, p<.001\right]$, and (3) the effect of consistency decreased as SOA increased $\left[F(3,84)=4.55, M S_{\mathrm{e}}=784, p<.01\right]$, with consistency effects of $45,28,16$, and $11 \mathrm{msec}$ at the four SOAs. Specific comparisons indicated that the consistency effect was highly reliable at the 100- and 350-msec SOAs ( $p<$ $.01)$, marginally reliable at the $700-\mathrm{msec} \operatorname{SOA}(p<.1)$, and not reliable at the $1,200-\mathrm{msec}$ SOA $(p>.15)$. The analogous ANOVA performed on percents correct yielded only a main effect of consistency, with consistent trials slightly but significantly more accurate than inconsistent ones $\left[99.6 \%\right.$ vs. $99.1 \%$ correct; $F(1,28)=4.92, M S_{\mathrm{e}}=$ $3.44, p<.05]$.

As in Experiment 1, the results also rule out the artifactual explanation of the consistency effect in terms of 

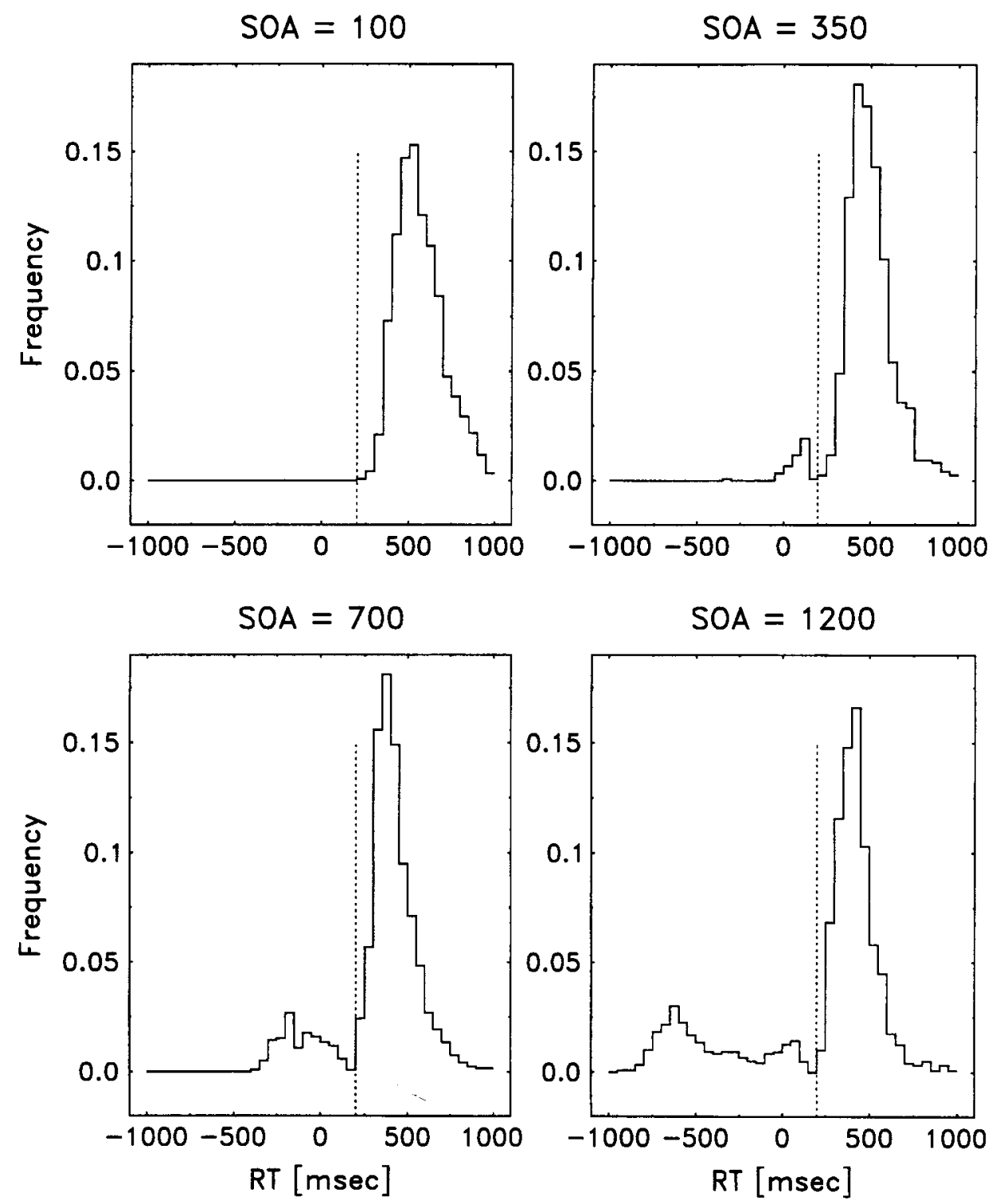

Figure 4. Observed frequency distributions of reaction times (RT) to probe tones at each stimulus onset asynchrony (SOA) in Experiment 2, pooling together trials with consistent and inconsistent probe tones (solid lines). Negative RTs indicate responses before tone onset. The dotted vertical line shows the 200-msec RT cutoff; responses faster than this cutoff were excluded in comparing the consistent and inconsistent conditions.

fast false alarms to the squares. First, Figure 6 shows that the large consistency effects at the shorter SOAs were not caused by a few very fast false alarms stretching the lower tail of the RT distribution in the consistent condition relative to the inconsistent one. Instead, the consistency effect is present throughout most or all of the RT distribution. Second, the consistency effect was again highly significant and slightly larger when errors were included in the analysis. Third, as shown in Figure 4, the frequency distributions of RTs to tones again suggested that the $200-\mathrm{msec}$ cutoff was effective in eliminating false alarms.
The decrease in the consistency effect with increasing SOA provides strong evidence that this effect arises from partial, rather than full, analysis of the nontarget square. The effect is large and highly significant at an SOA of $100 \mathrm{msec}$, where perceptual analysis of the nontarget square would virtually never be complete by the time the tone was presented, but small and nonsignificant at an SOA of 1,200 msec, where perceptual analysis would virtually always be completed before tone onset. This pattern is most easily explained by the assumption that priming is caused by information obtained during preliminary analysis of the square's size and that this prim- 
Table 2

Percentages of False Alarms for Which the Response Was Consistent or Inconsistent With Size Information at Each SOA in Experiment 2

\begin{tabular}{lcrrrr}
\hline & \multicolumn{5}{c}{ SOA (msec) } \\
\cline { 2 - 6 } & 100 & 350 & \multicolumn{1}{c}{700} & 1,200 & \multicolumn{1}{c}{$M$} \\
\hline Consistent & 0.00 & 4.00 & 12.33 & 22.25 & 9.65 \\
Inconsistent & 0.00 & 0.25 & 1.42 & 1.58 & 0.81 \\
Total & 0.00 & 4.25 & 13.75 & 23.83 & 10.46 \\
\hline
\end{tabular}

Note-A false alarm was defined as a response with RT less than $200 \mathrm{msec}$ relative to tone onset. The response was classified as consistent with the size information if it was made with the hand assigned to the target square of the more similar size; the response was classified as inconsistent if it was made with the other hand.

ing starts to dissipate after the square is determined to have a nontarget size. The preponderance of inconsistent trials might have helped cause the priming effect to dissipate. Because of this preponderance, full information about a nontarget square would suggest-at least on probabilistic grounds-that the inconsistent response should be prepared more than the consistent one. That the consistency effect did not actually reverse, as might have been expected, could suggest either that the longest SOA was not sufficient for such a reversal or that the priming mechanisms were not sufficiently sensitive to probabilities to behave as an ideal performer. A related study by Neely (1977) supports the latter interpretation; he found that automatic priming effects could be reversed within SOAs of $650 \mathrm{msec}$. Neely did not rely on an implicit adaptation to differential probabilities, however, but instead explicitly instructed his subjects to try to reverse the automatic priming on every trial. The comparison with Neely's study and the present results is extremely tentative, however, because Neely's study examined a type of semantic priming that is quite different from the response priming measured here.

\section{GENERAL DISCUSSION}

The two experiments reported here provide new evidence that is clearly at variance with the predictions of the ADC model suggested by Miller (1982). Specifically, the results indicate that partial information about a single stimulus attribute (i.e., the size of a square) is transmitted from perceptual to response processes, where it can have effects measurable with the probe-RT paradigm. Specifically, partial information facilitates responding to tones requiring the same responses most consistent with the partial information, relative to tones requiring the opposite response. This consistency effect is evidence that subjects carry out some response processing based on a partial size discrimination indicating that the stimulus is one of the larger two squares or one of the smaller two, before the exact size of the square has been determined.

The present evidence of response-level processing based on partial size information stands in contrast to previous results obtained with different paradigms indi- cating that partial information about a single attribute does not cause any of the types of response preparation examined previously (e.g., Miller, 1982, 1983; Miller \& Hackley, 1992). There are at least three possible explanations for these contrasting results, and further research will be required to decide the extent to which each is responsible for the contrast. One explanation is that the different paradigms are sensitive to different types of response processing, and these different types of response processing are, to some extent, dissociable. It appears that this explanation must be at least part of the story, because there is direct evidence that some types of response processing may take place and others may not take place, even on the very same experimental trials (Miller et al., 1996). At this point, for example, it appears likely that probe $\mathrm{RT}$ is sensitive to priming of responses at the responseselection stage, whereas other measures, such as the LRP, are sensitive to more peripheral motor preparation. If response priming is the only sort of preparation to occur using partial information in some cases (cf. Proctor \& Reeve, 1985; Reeve \& Proctor, 1984, 1985), then probe RT might be the only measure capable of revealing such response-level activity. A second possible explanation is that probe RT is simply more sensitive overall than previous measures. If so, previous null results might have been obtained simply because paradigms had insufficient statistical power to detect the response-level processing that actually did occur. This explanation does not seem very likely in the present case, however, because null results were obtained in two different paradigms using both RT and LRP measures (Miller, 1983; Miller \& Hackley, 1992), and because large and highly significant effects were obtained in those paradigms when response preparation could be based on partial information that uniquely specified a stimulus attribute. A third possibil-

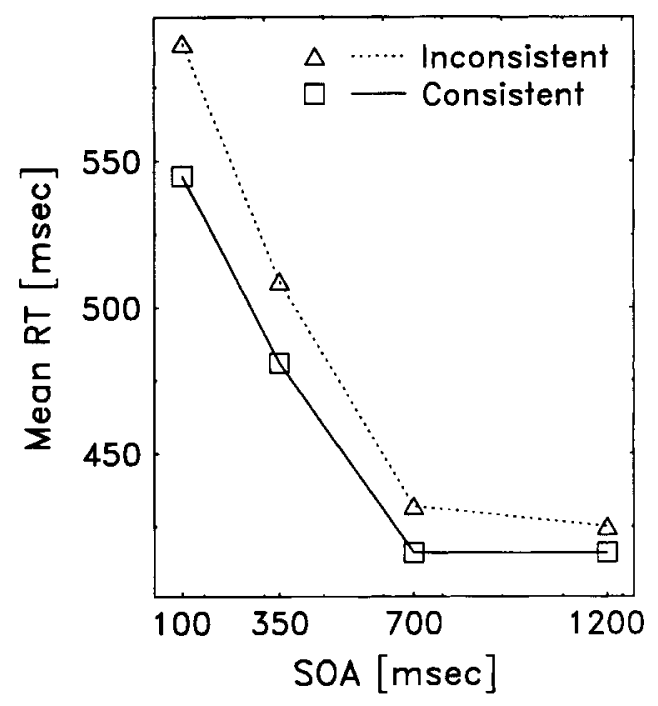

Figure 5. Mean reaction time ( $R T)$ to probe tones as a function of square consistency and square-to-tone stimulus onset asynchrony (SOA) in Experiment 2. 

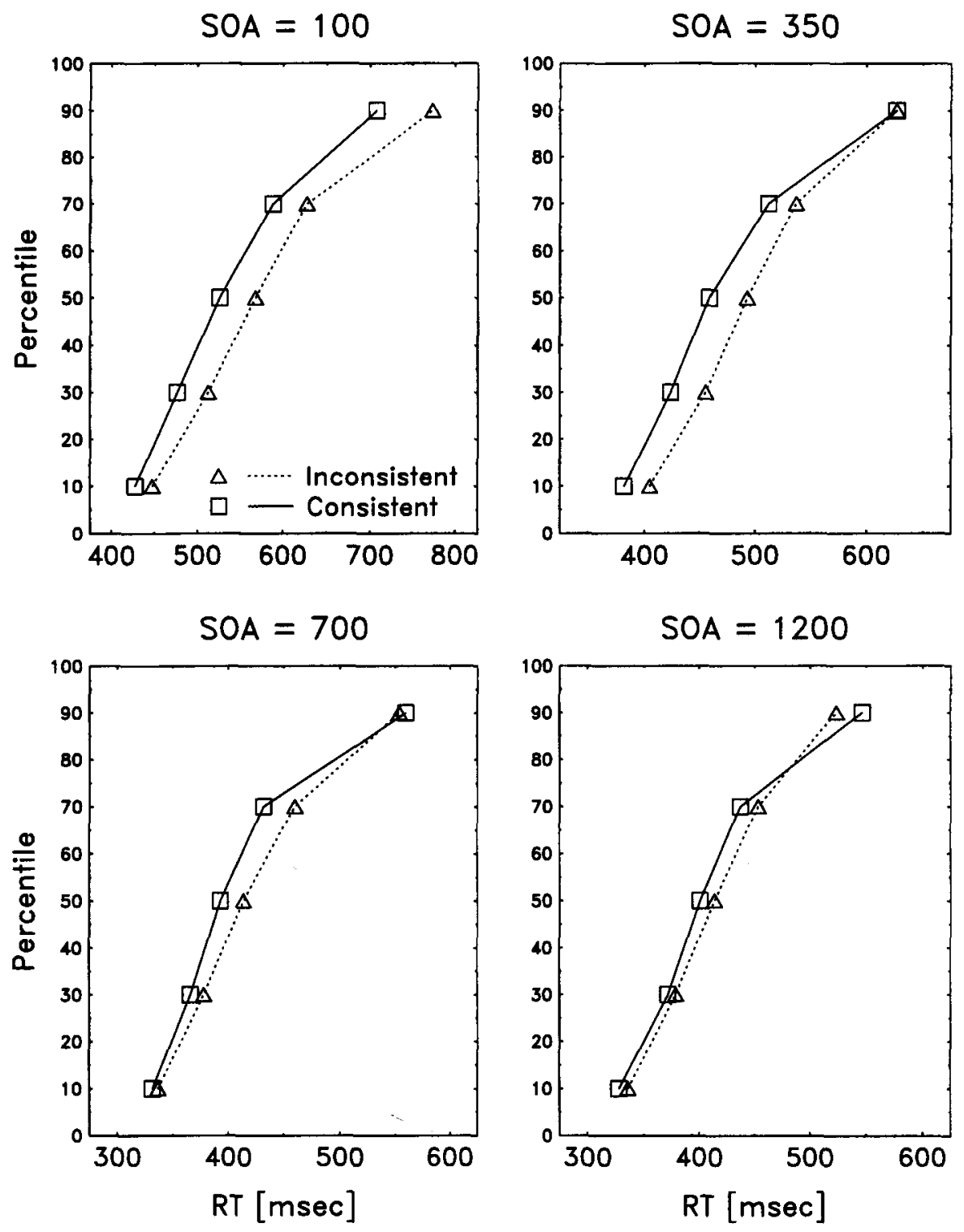

Figure 6. Vincentized cumulative distribution functions showing the mean reaction time (RT) to probe tones at each of five percentiles for consistent and inconsistent conditions at each stimulus onset asynchrony (SOA) in Experiment 2.

ity is that subtle differences in the experimental paradigms may influence the strategies used for stimulus identification and thereby change processing from discrete to continuous, or vice versa (cf. Coles, Smid, Scheffers, \& Otten, 1995; De Jong et al., 1988; Gratton et al., 1992). Although it seems quite likely that strategy differences play a role, this explanation is also unlikely to be the whole story. Miller et al. (1996) found evidence of response preparation using the probe-RT measure but not psychophysiological measures within the very same data set (i.e., same subjects and experimental trials); so this discrepancy cannot be attributed to a shift in strategy.

In combination with previous results, the present findings indicate that none of the models previously described (discrete, continuous, or ADC) adequately explains the temporal relationship of perceptual analysis and response preparation. Discrete models are incompatible with response preparation based on any type of partial information and are thus disconfirmed by both present and previous evidence (e.g., Miller \& Hackley, 1992) of such preparation. Continuous models predict that it should be possible to prepare responses based on any partial information about a stimulus; so they are compatible with the presence of response-level activity in the present experiments but not with the absence of more peripheral response preparation in previous studies with single-attribute stimuli (Miller, 1982, 1983; Miller \& Hackley, 1992). Finally, the ADC model predicts that re- 
sponse-level processing should occur if and only if partial information uniquely specifies a stimulus attribute, as has been observed. However, this model also suggests that no response processing of any kind should occur without specification of an attribute; thus, it is incompatible with the consistency effects observed in the present experiments.

The entire pattern of present and previous results seems to require a more complicated model than any previously considered. In particular, its main features seem to include (1) perceptual output about partially specified attributes as well as fully specified ones, (2) some response-level priming based on any available preliminary information, and (3) peripheral response preparation based only on preliminary information that fully specifies a stimulus attribute. Note that the model must incorporate a distinction between at least two different types of response processing, a distinction found in neither continuous nor ADC models as stated previously. At present, however, it is difficult to be more specific than this about the appropriate model, partly because of uncertainty about the nature of the two types of response-level processing that have been dissociated (priming and preparation). Following the analyses of Miller et al. (1996) and Rosenbaum (1985), for example, these might correspond to central selection of a motor program (i.e., response selection) and peripheral specification of free parameters within that program (i.e., response preparation). Perhaps, then, the system would use partial information within an attribute to begin the central selection process, but it might require complete attribute information to finish central selection and begin peripheral specification. Unfortunately, at this point, we cannot explain why partial information about an attribute can support response priming but not more peripheral preparation, whereas complete information can support both. One possibility is that partial information about an attribute only biases the response selection stage, but it does not produce a definitive decision of the sort that might be required for transmission to the response preparation stage. Another possibility is that response preparation is not carried out with singleattribute stimuli because the response preparation stage requires the involvement of a limited-capacity mechanism that is busy with other processing in tasks with these stimuli (see Band \& Miller, 1997; Ilan \& Miller, 1998).

Although the present results seem to require considerable extensions to the ADC model of the sort just described, there are two possibilities that might be developed to try to maintain the present version of the model. First, it could be argued that the consistency effect results entirely from processes taking place after recognition of the distractor is complete, not on partial information about the distractor (cf. Miller, 1985). It is difficult to explain, on this view, why an item known to be a distractor should favor one response or the other, but perhaps such an explanation could be developed. In addition, such an expla- nation does not seem promising in view of the fact that the consistency effect is obtained even at relatively short SOAs, at which distractor recognition would rarely be finished in the first place. Most importantly, this view seems completely ruled out by the results of Experiment 2, which showed faster consistent responses obtained even with a preponderance of inconsistent trials. In this case, subjects would almost certainly be encouraged to prepare the inconsistent response rather than the consistent one, after full information was available.

Second, as noted previously (Miller \& Hackley, 1992), the ADC model could be modified to handle such results by supposing that a sufficiently flexible information processing system would allow subjects to create and use a code for the partial information that is available (Miller \& Hackley, 1992). In the present experiments, for example, the subjects might have established a code for the concept of "one of the larger two squares" and another for "one of the smaller two squares." Discretely activating one of these codes would permit response preparation, and a subsequent exact discrimination of size, carried by a separate code, could indicate whether the response should be made or not. As Miller and Hackley pointed out, however, the ad hoc construction of codes to fit the model to experimental data is essentially circular. Even more importantly, this modification of the model does not address the dissociation between different measures of response-level processing: If subjects do form such codes, why isn't evidence of response preparation found in other measures besides the probe RT? Clearly, then, further research will be needed to specify the effects of different types of partial information on response-level processes.

\section{REFERENCES}

BAND, G. P. H., \& MilleR, J. O. (1997). Mental rotation interferes with response preparation. Journal of Experimental Psychology: Human Perception \& Performance, 23, 319-338.

Coles, M. G. H., Gratton, G., Bashore, T. R., Eriksen, C. W., \& DonCHIN, E. (1985). A psychophysiological investigation of the continuous flow model of human information processing. Journal of Experimental Psychology: Human Perception \& Performance, 11 , 529-553.

Coles, M. G. H., Smid, H. G. O. M., Scheffers, M. K., \& Otten, L. J. (1995). Mental chronometry and the study of human information processing. In M. D. Rugg \& M. G. H. Coles (Eds.), Electrophysiology of mind: Event-related brain potentials and cognition (pp. 86131). Oxford: Oxford University Press.

De Jong, R., Wierda, M., Mulder, G., \& Mulder, L. J. M. (1988). The use of partial information in response processing: A psychophysiological investigation. Journal of Experimental Psychology: Human Perception \& Performance, 14, 682-692.

Donders, F. C. (1969). On the speed of mental processes. In W. G. Koster (Ed.), Attention and performance II (pp. 412-431). Amsterdam: North-Holland. (Original work published 1868)

ERIKSEN, C. W., \& SChultz, D. W. (1979). Information processing in visual search: A continuous flow conception and experimental results. Perception \& Psychophysics, 25, 249-263.

Flowers, J. H. (1990). Priming effects in perceptual classification. Perception \& Psychophysics, 47, 135-148.

Gratton, G., Coles, M. G. H., \& Donchin, E. (1992). Optimizing the 
use of information: The strategic control of the activation of responses. Journal of Experimental Psychology: General, 121, 480-506.

Gratton, G., Coles, M. G. H., Sirevaag, E., ERiksen, C. W., \& DonchIN, E. (1988). Pre- and post-stimulus activation of response channels: A psychophysiological analysis. Journal of Experimental Psychology: Human Perception \& Performance, 14, 331-344.

HACKLEY, S. A., \& MiLler, J. O. (1995). Response complexity and precue interval effects on the lateralized readiness potential. Psychophysiology, 32, 230-241.

HOMmeL, B. (1993). The relationship between stimulus processing and response selection in the Simon task: Evidence for a temporal overlap. Psychological Research, 55, 280-290.

IlaN, A. B., \& Miller, J. O. (1998). On the temporal relations between memory scanning and response preparation. Journal of Experimental Psychology: Human Perception \& Performance, 24, 1501-1520.

LEVy, J., \& PASHLER, H. E. (1995). Does perceptual analysis continue during selection and production of a speeded response? Acta Psychologica, 90, 245-260.

LoFTUs, G. R. (1978). On interpretation of interactions. Memory \& Cognition, 6, 312-319.

MCClELLAND, J. L. (1979). On the time relations of mental processes: A framework for analyzing processes in cascade. Psychological Review, 86, 287-330.

Meyer, D. E., Osman, A. M., Irwin, D. E., \& Yantis, S. (1988). Modern mental chronometry. Biological Psychology, 26, 3-67.

Meyer, D. E., Yantis, S., Osman, A. M., \& Smith, J. E. K. (1985). Temporal properties of human information processing: Tests of discrete versus continuous models. Cognitive Psychology, 17, 445-518.

MiLLER, J. O. (1982). Discrete versus continuous stage models of human information processing: In search of partial output. Journal of Experimental Psychology: Human Perception \& Performance, 8, 273-296.

MILLER, J. O. (1983). Can response preparation begin before stimulus recognition finishes? Journal of Experimental Psychology: Human Perception \& Performance, 9, 161-182.

Miller, J. O. (1985). Discrete and continuous models of divided attention. In M. I. Posner \& O. S. M. Marin (Eds.), Attention and Performance XI: Mechanisms of attention (pp. 513-528). Hillsdale, NJ: Erlbaum.

MilLER, J. O. (1987). Evidence of preliminary response preparation from a divided attention task. Journal of Experimental Psychology: Human Perception \& Performance, 13, 425-434.

MiLleR, J. O. (1988). Discrete and continuous models of human information processing: Theoretical distinctions and empirical results. Acta Psychologica, 67, 191-257.

Miller, J. O., Coles, M. G. H., \& Chakraborty, S. (1996). Dissociation between behavioral and psychophysiological measures of response preparation. Acta Psychologica, 94, 189-208.

MiLleR, J. O., \& HACKLEY, S. A. (1992). Electrophysiological evidence for temporal overlap among contingent mental processes. Journal of Experimental Psychology: General, 121, 195-209.
Mulder, G., SMid, H. G. O. M., \& Mulder, L. J. M. (1992). On the transfer of partial information between perception and action. In D. E. Meyer \& S. Kornblum (Eds.), Attention and performance XIV: Synergies in experimental psychology, artificial intelligence, and cognitive neuroscience (pp. 567-588). Cambridge, MA: MIT Press.

NEELY, J. H. (1977). Semantic priming and retrieval from lexical memory: Roles of inhibitionless spreading activation and limited-capacity attention. Journal of Experimental Psychology: General, 106, 226-254.

Osman, A. M., Bashore, T. R., Coles, M. G. H., Donchin, E., \& MEYER, D. E. (1992). On the transmission of partial information: Inferences from movement-related brain potentials. Journal of Experimental Psychology: Human Perception \& Performance, 18, 217-232.

POSNER, M. I. (1978). Chronometric explorations of mind. Hillsdale, NJ: Erlbaum.

Proctor, R. W., \& Reeve, T. G. (1985). Compatibility effects in the assignment of symbolic stimuli to discrete finger responses. Journal of Experimental Psychology: Human Perception \& Performance, 11, 623-639.

REEVE, T. G., \& PROCTOR, R. W. (1984). On the advance preparation of discrete finger responses. Journal of Experimental Psychology: Human Perception \& Performance, 10, 541-553.

ReEve, T. G., \& Proctor, R. W. (1985). Nonmotoric translation processes in the preparation of discrete finger responses: A rebuttal of Miller's (1985) analysis. Journal of Experimental Psychology: Human Perception \& Performance, 11, 234-241.

Rosenbaum, D. A. (1985). Motor programming: A review and scheduling theory. In H. Heuer, U. Kleinbeck, \& K. H. Schmidt (Eds.), Motor behavior: Programming, control, and acquisition (pp. 1-33). Berlin: Springer-Verlag.

SMID, H. G. O. M., BöcKer, K. B. E., van TOUW, D. A., Mulder, G., \& BRUNIA, C. H. M. (1996). A psychophysiological investigation of the selection and the use of partial stimulus information in response choice. Journal of Experimental Psychology: Human Perception \& Performance, 22, 3-24.

STERNBERG, S. (1969). The discovery of processing stages: Extensions of Donders' method. Acta Psychologica, 30, 276-315.

\section{NOTES}

1. We use the term stage in the general sense of a functionally distinct process, without intending to imply that these necessarily operate in strict succession.

2. We are indebted to Robert Proctor and an anonymous reviewer for their helpful suggestions with respect to this artifact.

(Manuscript received July 10, 1996; revision accepted for publication November 24, 1997.) 